\title{
Coprecipitation Equilibrium of Lanthanoid(III) Ions with Calcium Oxalate
}

\author{
Yoshihiro Iwata, Hisanori Imura and Nobuo SuzukI ${ }^{\dagger}$ \\ Department of Chemistry, Faculty of Science, Tohoku University, Sendai 980, Japan
}

\begin{abstract}
The coprecipitation equilibrium of lanthanoid( $\mathrm{Ln}(\mathrm{III})$ ) ions such as $\mathrm{La}(\mathrm{III}), \mathrm{Ce}(\mathrm{III}), \mathrm{Nd}(\mathrm{III}), \mathrm{Sm}(\mathrm{III}), \mathrm{Tb}$ (III), $\mathrm{Tm}$ (III) and $\mathrm{Lu}$ (III) with calcium oxalate was investigated by a radiotracer method. The distribution ratio of $L$ (III) between the solid phase of calcium oxalate and the aqueous phase containing trace amounts of $\operatorname{Ln}$ (III) ion was determined. It was found that the coprecipitation mechanism is an ion exchange of three $\mathrm{Ca}$ (II) ions with two $\mathrm{Ln}(\mathrm{III})$ ions. The equilibrium constants, $K=\left[\mathrm{Ln}^{3+}\right]_{\text {solid }}^{2}\left[\mathrm{Ca}^{2+}\right]_{\text {aq }}^{3} /\left[\mathrm{Ln}^{3+}\right]_{\text {aq }}^{2}$, were determined for these 7 lanthanoid ions.
\end{abstract}

Keywords Coprecipitation, lanthanoid, calcium oxalate, equilibrium constant

It is known that lanthanoid ions in a solution containing oxalate form a fairly insoluble precipitate of lanthanoid oxalate ${ }^{1}$, and that the oxalate precipitation has often been used in the gravimetric analysis of lanthanoid(III). Traces of lanthanoid(III) ions are coprecipitated with calcium oxalate, and this reaction has been used as a preseparation and preconcentration method for the determination of trace lanthanoid(III) by spectrophotometry ${ }^{2}$, atomic absorption spectrometry ${ }^{3}$ and neutron activation analysis. ${ }^{4}$ However, the coprecipitation mechanism of lanthanoid(III) with calcium oxalate has scarcely been investigated.5,6 It was observed that the distribution of the lanthanoid(III) between the aqueous phase and the solid surface by homogeneous coprecipitation with dimethyloxalate ${ }^{5}$, and adsorption onto a calcium oxalate crystal. ${ }^{6}$ And the logarithmic distribution coefficients ${ }^{5}$ and distribution coefficients between the aqueous phase and the solid surface were presented. ${ }^{6}$ It was also suggested that a replacement of three calcium ions by two lanthanoid ions occurred. However, there has been no research work to find a true thermodynamic equilibrium constant of the calcium-lanthanoid system.

In the present study the coprecipitation equilibrium of seven lanthanoid(III) ions such as lanthanum(III), cerium(III), neodymium(III), samarium(III), terbium(III), thulium(III) and lutetium(III) with calcium oxalate was studied. The distribution equilibrium of the lanthanoid(III) ions between the calcium oxalate phase and the aqueous phase was achieved by long-term stirring of a solution containing calcium oxalate. The equilibrium constants of the coprecipitation were also determined; the present fundamental study should be very efficient for predicting the most appropriate

† To whom correspondence should be addressed. condition of the selective coprecipitation of traces of lanthanoid(III).

\section{Experimental}

\section{Reagents}

Oxalic acid dihydrate was of guaranteed reagent grade and recrystallized from water. A stock solution of oxalic acid $(0.40 \mathrm{M})$ was standardized by acid-base titration with sodium carbonate. A stock solution of calcium(II) $(1.0 \mathrm{M})$ was prepared by dissolving calcium nitrate of guaranteed reagent grade in water and standardized by chelate titration with ethylenediaminetetraacetate. Standard solutions of lanthanoid(III) such as lanthanum(III), neodymium(III), samarium(III), terbium(III), thulium(III) and lutetium(III) were prepared by dissolving a known amount of each oxide (more than $99.9 \%$ purity) in nitric acid and diluting with water. Cerium(IV) oxide ( $99.9 \%$ purity) was dissolved in a $6 \mathrm{M}$ hydrochloric acid solution containing hydrogen peroxide $(5 \%)$. It was evaporated to dryness, dissolved in hydrochloric acid, and diluted with water. All lanthanoid oxides were treated by heating at $800^{\circ} \mathrm{C}$ for $3 \mathrm{~h}$ prior to prepare the corresponding standard solutions.

Radioactive tracers $\left({ }^{140} \mathrm{La},{ }^{141} \mathrm{Ce},{ }^{147} \mathrm{Nd},{ }^{153} \mathrm{Sm},{ }^{160} \mathrm{~Tb}\right.$, ${ }^{170} \mathrm{Tm}$ and ${ }^{177} \mathrm{Lu}$ ) were produced by neutron irradiation of the corresponding oxide or nitrate in a nuclear reactor (JRR-4) of Japan Atomic Energy Research Institute at thermal neutron flux of $5.5 \times 10^{13} \mathrm{n} \mathrm{cm}^{-2} \mathrm{~s}^{-1}$ for $12 \mathrm{~h}$. Irradiated targets were dissolved in either nitric acid or hydrochloric acid. A radioactive working solution of lanthanoid(III) was prepared by mixing an appropriate amount of the standard solution of lanthanoid(III) with the radioactive tracer solution, evap- 
orating to dryness, and dissolving in $0.01 \mathrm{M}$ nitric acid. Acids and aqueous ammonia were of high purity and water was doubly distilled. Other reagents were of guaranteed reagent grade.

\section{Procedure}

A 5-ml portion of $0.05-0.30 \mathrm{M}$ calcium(II) solution containing $2.0 \times 10^{-7}-6.0 \times 10^{-5} \mathrm{M}$ radioactive lanthanoid(III) was mixed with 5-ml of the $1.0 \times 10^{-3} \mathrm{M}$ oxalic acid solution containing $2.0 \times 10^{-3} \mathrm{M}$ chloroacetic acid as a $\mathrm{pH}$ buffer. The $\mathrm{pH}$ was adjusted to $3.0 \pm 0.1$ with aqueous ammonia and nitric acid, and the ionic strength was adjusted to $0.50 \mathrm{M}$ with ammonium nitrate. Though, immediately after mixing, the formation of a white precipitate was observed, the mixture was stirred for more than $7 \mathrm{~d}$ in a thermostated water bath at $25 \pm 0.1^{\circ} \mathrm{C}$ and finally centrifuged at $3000 \mathrm{rpm}$ for $5 \mathrm{~min}$. The $\gamma$-radioactivity of the supernatant was measured using a well-type $\mathrm{NaI}(\mathrm{Tl})$ scintillation detector connected with a single-channel analyzer. The equilibrium $\mathrm{pH}$ value of the solution was measured with a glass electrode. The distribution ratio $(D)$ of lanthanoid(III) defined as the ratio of the concentration of lanthanoid(III) in the solid phase ( $\left.\mathrm{mol} \mathrm{kg}^{-1}\right)$ to that in the aqueous phase $\left(\mathrm{mol} \mathrm{dm}^{-3}\right)$ was calculated by

$$
D=\frac{\left(A_{\mathrm{i}}-A_{\mathrm{aq}}\right)}{A_{\mathrm{aq}}} \cdot \frac{v}{m} .
$$

where $A_{\mathrm{i}}$ is the initial radioactivity of the aqueous phase; $A_{\text {aq }}$ is the radioactivity of the supernatant after coprecipitation, and $v$ and $m$ are the volume of aqueous phase $\left(\mathrm{dm}^{3}\right)$ and the weight of solid phase $(\mathrm{kg})$, respectively. In order to ascertain the complete phase separation by centrifugation, the supernatant obtained after centrifugation was filtered with a membrane filter (pore size: $0.45 \mu \mathrm{m}$, acetylcellulose); the radioactivity of the filtrate was then measured. No radioactivity change was observed either before and after filtration.

\section{Results and Discussion}

The distribution equilibrium of lanthanoid(III) between the solid phase of calcium oxalate and the aqueous phase containing lanthanoid(III) was examined. Calcium oxalate was prepared by mixing of $1.0 \mathrm{M}$ calcium chloride with $1.0 \mathrm{M}$ oxalic acid under vigorous stirring, then filtered and dried at $105^{\circ} \mathrm{C}$ for $2 \mathrm{~h}$. A $100-\mathrm{mg}$ portion of calcium oxalate was added to an aqueous phase containing $1.0 \times 10^{-6} \mathrm{M}$ terbium(III) and $0.10 \mathrm{M}$ sodium perchlorate at $\mathrm{pH} 2.4$ and stirred at $25 \pm 0.1^{\circ} \mathrm{C}$. As Fig. 1 shows, though terbium(III) readily distributes to the solid phase, the distribution ratio gradually increases with the stirring time. It seems that the distribution equilibrium cannot be achieved under the given conditions. A similar tendency is observed by a distribution of terbium(III) coprecipitated in the calcium oxalate to a fresh aqueous phase not containing

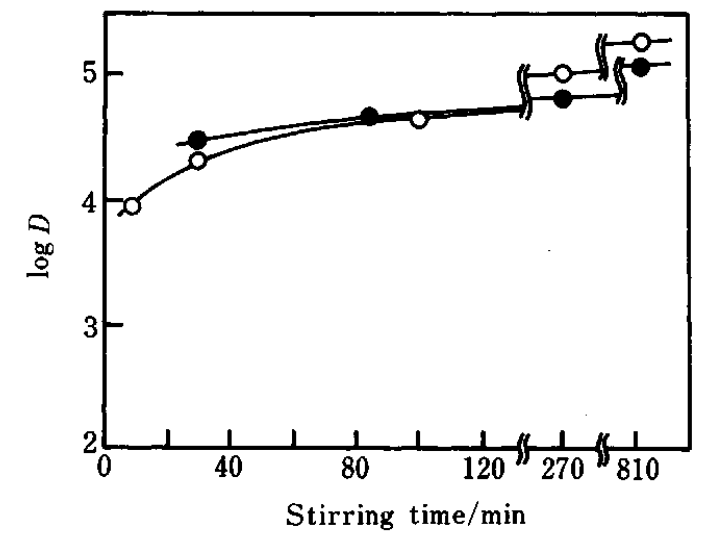

Fig. 1 Effect of the stirring time on the adsorption of terbium(III) on the solid calcium oxalate. Initial concentration of terbium(III) is $1.0 \times 10^{-6} \mathrm{M}$. O: $1.0 \times 10^{-6} \mathrm{M}$ $\mathrm{Tb}(\mathrm{III})$ solution was stirred with fresh solid phase.

$\mathrm{Tb}(\mathrm{III})$ adsorbed solid phase was stirred with fresh aqueous phase.

terbium(III). Also in this case (Fig. 1), the distribution ratio of terbium(III) gradually increases with the stirring time. These results suggest that terbium(III) distributed onto the surface of calcium oxalate is gradually transferred into the inner lattice of calcium oxalate, accompanied by a recrystallization of calcium oxalate. Hence, the overall coprecipitation occurs through two processes: the distribution of lanthanoid(III) onto the surface of calcium oxalate precipitate and the recrystallization of the precipitate through a transferring of the adsorbed lanthanoid into the calcium oxalate crystal; it takes long time to reach complete equilibrium.

The coprecipitation equilibration between the aqueous phase and the solid phase was examined as follows: to the aqueous phase containing $1.0 \times 10^{-6} \mathrm{M}$ lanthanoid(III) and $0.025 \mathrm{M}$ or $0.15 \mathrm{M}$ calcium(II), $5.0 \times 10^{-4} \mathrm{M}$ oxalic acid was added and stirred for $7-13 \mathrm{~d}$ at $25 \pm 0.1^{\circ} \mathrm{C}$. This is a so-called substoichiometric precipitation of carrier calcium using a deficient amount of oxalic acid, and is a very effective experimental condition for examining the coprecipitation isotherm in order to avoid an extremely high distribution of lanthanoid by using a great excess amount of precipitant, as in the usual coprecipitation experiments. Figure 2 shows that the distribution ratio of lanthanum(III), terbium(III) and lutetium(III) becomes constant after more than $9 \mathrm{~d}$ of stirring, and that the overall coprecipitation equilibrium is achieved. Figure 2 also shows that the distribution ratio of lanthanoid(III) at a higher concentration of calcium ion is low compared with the case using a lower concentration of calcium ions. This phenomenon seems to be a competitive reaction between lanthanoid(III) and excess calcium(II) against a substoichiometric amount of oxalate ions. In the absence of a calcium carrier, no loss or no precipitation of lanthanoid(III) was observed 


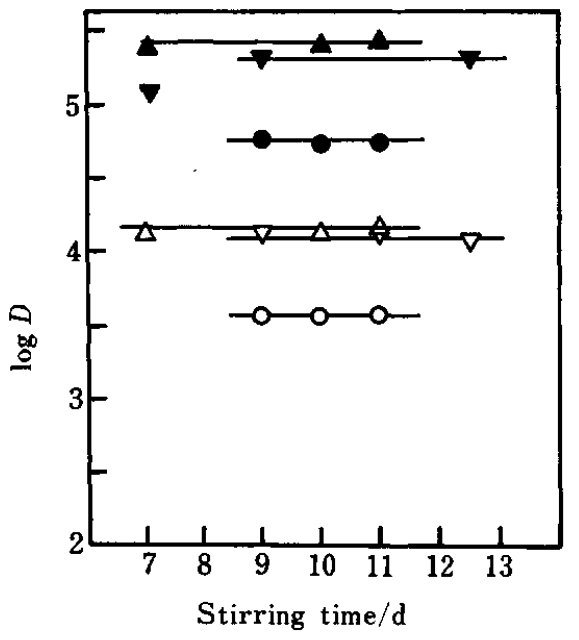

Fig. 2 Effect of the stirring time on the coprecipitation of lanthanoid(III) with calcium oxalate. Initial concentration of calcium(II): filled symbols, $0.025 \mathrm{M}$; open symbols, $0.15 \mathrm{M}$. Initial concentration of oxalic acid, $5.0 \times 10^{-4} \mathrm{M}$. OO, $\mathrm{La}(\mathrm{III})$; $\Delta \mathbf{\Lambda}, \mathrm{Tb}(\mathrm{III}) ; \nabla \nabla, \mathrm{Lu}(\mathrm{III})$.

upon the addition of an oxalate solution.

The following coprecipitation equilibrium based on the ion exchange mechanism may be proposed:

$$
2 \mathrm{Ln}^{3+}+3 \overline{\mathrm{Ca}}^{2+} \rightleftharpoons 2 \overline{\mathrm{Ln}}^{3+}+3 \mathrm{Ca}^{2+},
$$

where the bar over the ions denotes the solid phase. The equilibrium constant, $K^{\circ}$, is defined as

$$
K^{\circ}=\frac{\left[\overline{\mathbf{L n}}^{3+}\right]^{2}\left[\mathrm{Ca}^{2+}\right]^{3}}{\left[\mathbf{L n}^{3+}\right]^{2}\left[\overline{\mathbf{C a}}^{2+}\right]^{3}} \cdot \frac{\bar{\gamma}_{\mathrm{Ln} 3+}^{2} \gamma_{\mathrm{C} 2+}^{3}}{\gamma_{\mathrm{Ln} 3+}^{2} \bar{\gamma}_{\mathrm{Ca} 2+}^{3}} .
$$

In the assumption that the activity of calcium(II) in the solid phase equals unity and that the activity coefficients of ions, $\gamma$, in the both phases are constant under the present experimental condition, the apparent equilibrium constant, $K$, is

$$
K=\left[\overline{\operatorname{Ln}}^{3+}\right]^{2}\left[\mathrm{Ca}^{2+}\right]^{3} /\left[\mathrm{Ln}^{3+}\right]^{2} .
$$

When the distribution ratio of lanthanoid(III) is simply expressed as $D=\left[\overline{\mathbf{L n}^{3+}}\right] /\left[\mathrm{Ln}^{3+}\right]$ the following equation is derived:

$$
\log D=-\frac{3}{2} \log \left[\mathrm{Ca}^{2+}\right]+\frac{1}{2} \log K \text {. }
$$

When the complexation of lanthanoid(III) with a dominant anion present in the aqueous phase (nitrate ion) is taken into account, Eq. (5) can be rewritten using the formation constant of nitrate complexes of lanthanoid(III), $\beta_{n}$, as

$$
\begin{aligned}
\log D= & -\frac{3}{2} \log \left[\mathrm{Ca}^{2+}\right]-\log \left(1+\Sigma \beta_{n}\left[\mathrm{NO}_{3}^{-}\right]^{n}\right) \\
& +\frac{1}{2} \log K .
\end{aligned}
$$

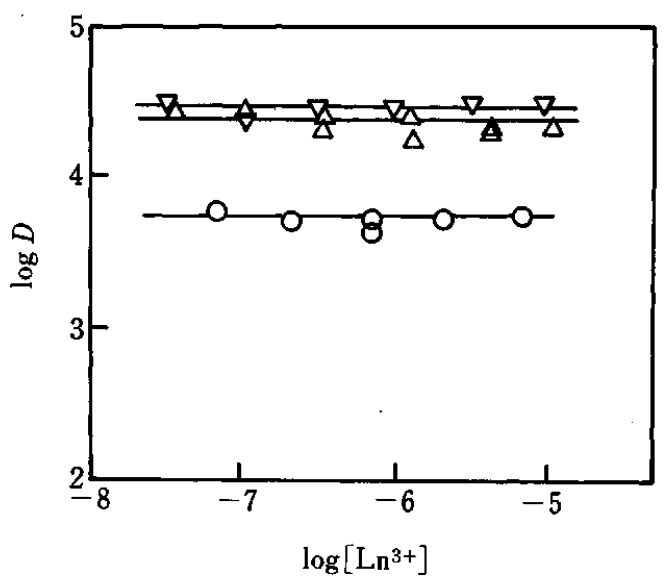

Fig. 3 Coprecipitation of various concentrations of lanthanoid(III) with calcium oxalate. Initial concentration of $\mathrm{Ca}(\mathrm{II})$, 0.10 M. O, La(III); $\Delta$, Tb(III); $\nabla, \mathrm{Lu}(\mathrm{III})$.

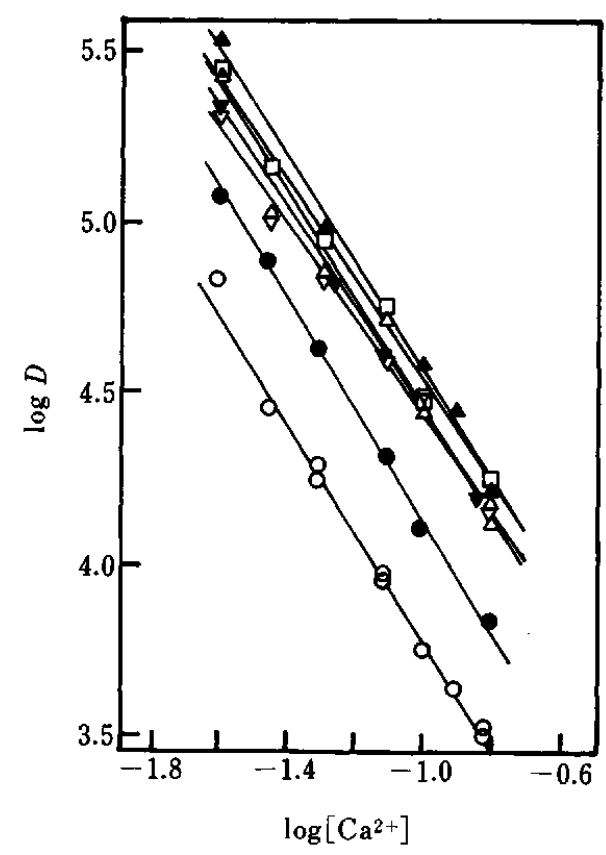

Fig. 4 Effect of the calcium(II) concentration on the distribution ratio of lanthanoid(III). Initial concentration of lanthanoid(III): $1.0 \times 10^{-6} \mathrm{M}$. O, La(III);, $\mathrm{Ce}(\mathrm{III}) ; \Delta$, $\mathrm{Nd}(\mathrm{III}) ; \square, \mathrm{Sm}(\mathrm{III}) ; \Delta, \mathrm{Tb}(\mathrm{III}) ; \nabla, \mathrm{Tm}(\mathrm{III}) ; \nabla, \mathrm{Lu}(\mathrm{III})$.

Equations (5) and (6) indicate that the distribution ratio of lanthanoid(III) does not depend on the lanthanoid concentration but, rather, on the calcium concentration in the aqueous phase.

Figure 3 shows the effect of the lanthanoid(III) concentration on the distribution ratio of lanthanoid(III). As is expected, the distribution ratio of lanthanum(III), terbium(III) and lutetium(III) is constant over a wide of lanthanoid(III) concentrations from $3 \times 10^{-8}$ to $1 \times 10^{-5} \mathrm{M}$. Figure 4 shows the effect of the calcium(II) concentration in the aqueous phase on the distribution ratio of lanthanoid(III). The $\log D$ linearly decreases with an increase in the calcium(II) 
Table 1 Equilibrium constant for the coprecipitation of lanthanoid(III) with calcium oxalate at $25^{\circ} \mathrm{C}$

\begin{tabular}{cccccc}
\hline Ln(III) & Slope $^{\mathrm{a}}$ & $\log K^{\mathrm{a}}$ & Slope $^{\mathrm{b}}$ & $\log K^{\mathrm{b}}$ & $(n)$ \\
\hline $\mathrm{La}$ & -1.6 & $4.56 \pm 0.06$ & -1.6 & $4.93 \pm 0.09$ & $(10)$ \\
$\mathrm{Ce}$ & -1.6 & $5.30 \pm 0.07$ & -1.6 & $5.68 \pm 0.11$ & $(6)$ \\
$\mathrm{Nd}$ & -1.6 & $6.15 \pm 0.10$ & -1.6 & $6.62 \pm 0.12$ & $(5)$ \\
$\mathrm{Sm}$ & -1.4 & $6.10 \pm 0.09$ & -1.5 & $6.64 \pm 0.08$ & $(6)$ \\
$\mathrm{Tb}$ & -1.5 & $5.92 \pm 0.16$ & -1.5 & $6.22 \pm 0.16$ & $(6)$ \\
$\mathrm{Tm}$ & -1.4 & $5.90 \pm 0.09$ & -1.5 & $6.06 \pm 0.08$ & $(5)$ \\
$\mathrm{Lu}$ & -1.4 & $5.80 \pm 0.12$ & -1.4 & $6.00 \pm 0.11$ & $(6)$ \\
\hline
\end{tabular}

a. Based on Eq. (5).

b. Based on Eq. (6) using the literature values of $\beta_{1}$ for $\mathrm{Ln}\left(\mathrm{NO}_{3}\right)^{2+}$ : La, 1.37; $\mathrm{Ce}, 1.3^{7} ; \mathrm{Nd}, 1.7^{8} ; \mathrm{Sm}, 2.1^{9} ; \mathrm{Tb}, 0.94^{9}$; $\mathrm{Tm}, 0.56^{10} ; \mathrm{Lu}, 0.6^{7}$.

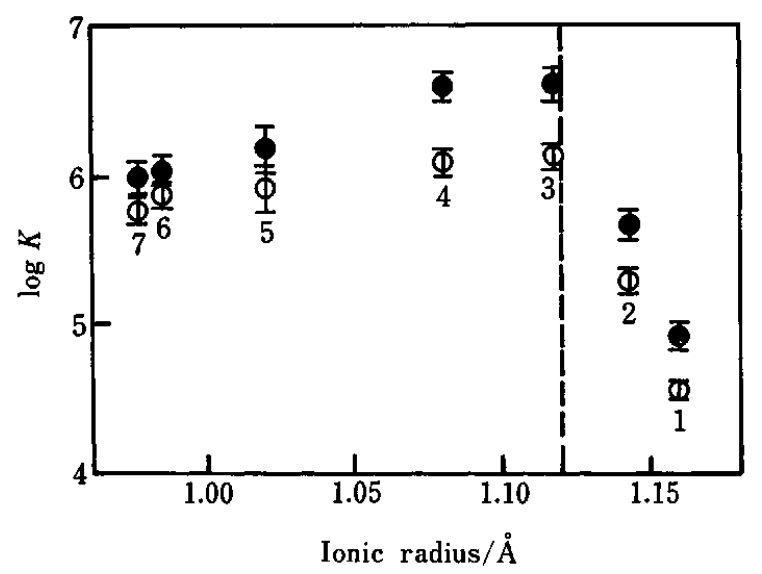

Fig. 5 Relationship between the coprecipitation equilibrium constant of lanthanoid(III) with calcium oxalate, $K$, and the ionic radius of lanthanoid(III). The vertical broken line indicates the ionic radius of $\mathrm{Ca}$ (II) (see the text). Open symbols, $K$ calculated by Eq. (5); filled symbols, $K$ by Eq. (6). 1, La(III); 2, Ce(III); 3, Nd(III); 4, Sm(III); 5, Tb(II); 6, Tm(III); 7, Lu(III).

concentration in the aqueous phase. The slope of the straight lines lies between -1.4 and -1.6 , which is in good agreement with a slope of -1.5 expected from Eqs. (5) and (6). Table 1 summarizes the slope of the straight line observed in Fig. 4 and the equilibrium constant based on Eqs. (5) and (6). In Table 1 the contribution of nitrate complex was taken into account in order to calculate the equilibrium constant by Eq. (6) using literature values of $\beta_{1}{ }^{7-10}$ The $\log K$ values calculated using Eq. (5) are close to those calculated using Eq. (6). These results suggest that the coprecipitaion mechanism of trace lanthanoid(III) with calcium oxalate is an ion exchange reaction between three calcium(II) ions and two lanthanoid(III) ions in the calcium oxalate phase.

Figure 5 shows an interesting relationship between the equilibrium constants of the coprecipitation reaction, $K$, and the ionic radii of lanthanoid(III) ions. Since the coordination number of calcium(II) in calcium oxalate is known to be eight by X-ray crystallography ${ }^{11}$, the ionic radii of lanthanoid(III) ions in the crystal must also adopt a coordination number of eight. ${ }^{12}$ The $\log K$ obtained by Eq. (5) as well as Eq. (6) increase with an increase in the ionic radius of lanthanoid(III) up to neodymium(III), which has the closest ionic radius to that of calcium(II)(1.12 $\AA$ ). ${ }^{12}$ For the lighter lanthanoid(III) ions having a larger ionic radius than that of calcium(II), i.e., lanthanum(III) and cerium(III), $\log K$ decrease with an increase in the ionic radius of lanthanoid(III). These results suggest that the exchange reaction between the calcium(II) ion and the lanthanoid(III) ion takes place at the site of the calcium ion in the calcium oxalate crystal.

Usually, in analytical separation, the coprecipitation of trace metals with a host precipitate is made by complete and quantitative precipitation of the host ions by using a great excess amount of the precipitant; however, by considering the large values of the coprecipitation equilibrium constants determined here, we can predict from Eq. (5) the most satisfactory condition to completely coprecipitate trace lanthanoid with calcium oxalate. This is very valuable for developing a more effective and selective separation method for actual trace lanthanoid(III) samples. ${ }^{13}$

This work was supported by a Grant-in-Aid for Scientific Research from the Ministry of Education, Science and Culture of Japan. The authors wish to express their thanks to the Japan Atomic Energy Research Institute, and the Radioisotope Center, Tohoku University, for their helpful cooperation.

\section{References}

1. L. G. Sille'n and A. E. Martell, "Stability Constants of Metal-ion Complexes", Supplement No. 1, Section I, p. 247, The Chemical Society, London, 1971.

2. V. G. Goryushina, S. B. Savvin and E. V. Romanova, Zh. Anal. Khim., 18, 1340 (1963).

3. J. G. S. Gupta, Talanta, 31, 1053 (1984).

4. T. Oda, Radioisotopes, 18, 39 (1969).

5. M. Matsui, Bull. Chem. Soc. Jpn., 39, 1114 (1966).

6. E. M. Pazukhin and R. V. Bryzgalova, Zh. Obshch. Khim., 49, 15 (1979).

7. D. F. Peppard, G. W. Mason and I. Hucher, J. Inorg. Nucl. Chem., 24, 881 (1962).

8. A. I. Mikhailichenko and I. E. Kurdin, Radiokhimiya, 11, $356(1969)$.

9. Z. Kolarik, Collect. Czech. Chem. Commun., 32, 435 (1967).

10. G. R. Choppin and W. F. Strazik, Inorg. Chem., 4, 1250 (1965).

11. S. Deganello and O. E. Piro, N. Jb. Miner. Mh., 1981, 81.

12. R. D. Shannon, Acta Crystallogr., A32, 751 (1976).

13. Y. Iwata, H. Imura and N. Suzuki, Proceedings of 37th Annual Meeting of The Japan Society for Analytical Chemistry, Sapporo, October 1988, p. 946.

(Received February 13, 1990) (Accepted July 30, 1990) 